\title{
Mobile software prototype - Patient guidance for laboratory investigation preparations
}

\author{
Dr. E.P.D.S. Ediriweera \\ Postgraduate Institute of Medicine, University of Colombo, Sri Lanka. \\ Dr. P.E.K.B. Ranatunga \\ Postgraduate Institute of Medicine, University of Colombo, Sri Lanka. \\ Dr. B.D.W. Jayamanne \\ Postgraduate Institute of Medicine, University of Colombo, Sri Lanka. \\ Contact e-mail address: edileepa@gmail.com \\ eHealth Sri Lanka 2010,1(suppl.1):S39 \\ DOI: http://dx.doi.org/10.4038/sljbmi.v1i0.3692
}

Only the Abstract is available

\begin{abstract}
Awareness of health among general public is on the increase with the advancement of information and communication technology. The speed at which the required information is delivered to the client, plays a major role in such a system. Recent advancement of mobile technology and wide spread usage make life easier regarding this aspect, especially with the Short Message Service (SMS). Obtaining preparation instructions for investigations is useful to the client, so they can go to the laboratories prepared. eg. Lipid Profile - 14 hours of fasting. Medical Practitioners also can revise the awareness for preparation of uncommon investigations, reference range and their availability at his closest reach. This will automatically produces an island-wide database of registered laborites. In addition we can expect standardisation of prices for common investigations will also happen with the time. Proposed system provides instructions to the client on preparation for laboratory investigations which can be helpful both to the patients and the medical practitioners. Further expansion of the system will provide the list of locally available laboratories in which the particular investigation can be done and the relevant reference range. Client is expected to send an SMS giving the list of investigations. The system will match the investigations with a database containing investigations and relevant preparation instructions. Then reply via SMS to the client with this information. The database will be constructed and maintained using national guidelines.

Keywords - Mobile software prototype, registered laborites, common investigations
\end{abstract}

